\title{
44381 - PAIN EASE VAPOCOOLANT SPRAY VERSUS PLACEBO IN REDUCING PAIN ASSOCIATED WITH INTRAVENOUS INSERTION IN CHILDREN: RCT
}

\author{
William Splinter, University of Ottawa, Ottawa, ON, Canada; \\ Karen Splinter, University of Ottawa; \\ Ken Farion, University of Ottawa; \\ K Newhook, University of Ottawa; \\ S Tan, University of Ottawa; \\ J Anderson, University of Ottawa; \\ I Gaboury, Chalmers Research Group;
}

INTRODUCTION: Intravenous (IV) insertion is a distressing experience for many children. Topical anesthetic agents have been developed to reduce the pain of IV insertion, but the application time required for good effect makes them often impractical in the Emergency Department (ED). Pain Ease is a vapocoolant spray that quickly numbs the skin to reduce the conduction of pain stimuli. Our Objective was to assess the efficacy of Pain Ease in reducing the pain associated with IV insertion in children.

METHODS: Local IRB approval was obtained for this study. This double-blind, blockrandomized controlled trial compared Pain Ease spray to placebo spray concealed in sequentially numbered opaque envelopes. A convenience sample of children 6-12 yrs requiring urgent (not emergent) IV's in the ED rated their pre-procedure anxiety on a $10 \mathrm{~cm}$ visual analogue scale (VAS). One of two Child Life Specialists (CLS) provided patient preparation and distraction during the procedure. The treating Registered Nurse $(\mathrm{RN})$ chose and prepped the insertion site (dorsum of the hand or antecubital fossa). The research assistant then sprayed the treatment product and the IV attempt was made within 60 seconds. Patients rated the pain of insertion using a $10 \mathrm{~cm}$ VAS. The parent, RN and CLS rated the child's pain and recorded which group they felt the child was randomized to.

RESULTS: Eighty children were randomized and completed the trial. Baseline characteristics and pre-procedure anxiety scores were similar between groups. Pain with IV insertion was significantly less with Pain Ease (mean VAS 3.69 vs 5.61, p=0.005). The results remained significant when adjusted for needle size $(\mathrm{p}=0.009)$. Successful cannulation on 1 st attempt was significantly higher with Pain Ease ( $85 \%$ vs $63 \%, \mathrm{p}=0.026)$. The parent, RN and CLS all rated the child s pain as less with Pain Ease ( $\mathrm{p}=0.04, \mathrm{p}=0.012, \mathrm{p}=0.003$ respectively). Only the CLS's were able to correctly identify the treatment group more often than expected by chance $(\mathrm{p}<0.001)$.

DISCUSSION: Pain Ease quickly and effectively reduces pain associated with IV insertion in children and improves success of IV insertion. 